\title{
Mechanics of individual isolated vortices in a cuprate superconductor
}

\author{
Ophir M. Auslaender ${ }^{1 \star}$, Lan Luan ${ }^{1}$, Eric W.J. Straver ${ }^{1}$, Jennifer E. Hoffman ${ }^{1,2}$, Nicholas C. Koshnick', \\ Eli Zeldov ${ }^{1,3}$, Douglas A. Bonn ${ }^{4}$, Ruixing Liang ${ }^{4}$, Walter N. Hardy ${ }^{4}$ and Kathryn A. Moler ${ }^{1}$
}

\begin{abstract}
Superconductors often contain quantized microscopic whirlpools of electrons, called vortices, that can be modelled as one-dimensional elastic objects ${ }^{1}$. Vortices are a diverse area of study for condensed matter because of the interplay between thermal fluctuations, vortex-vortex interactions and the interaction of the vortex core with the three-dimensional disorder landscape ${ }^{2-5}$. Although vortex matter has been studied extensively ${ }^{1,6,7}$, the static and dynamic properties of an individual vortex have not. Here, we use magnetic force microscopy (MFM) to image and manipulate individual vortices in a detwinned $\mathrm{YBa}_{2} \mathrm{Cu}_{3} \mathrm{O}_{6.991}$ single crystal, directly measuring the interaction of a moving vortex with the local disorder potential. We find an unexpected and marked enhancement of the response of a vortex to pulling when we wiggle it transversely. In addition, we find enhanced vortex pinning anisotropy that suggests clustering of oxygen vacancies in our sample and demonstrates the power of MFM to probe vortex structure and microscopic defects that cause pinning.
\end{abstract}

A superconducting vortex is characterized by two length scales: the nanoscale core size and the much larger magnetic penetration depth, $\lambda$. Pinning can occur when the core is co-located with a defect that locally suppresses superconductivity. $\lambda$ determines the decay length for the currents encircling the core and the elastic properties of a vortex. Here, we probe the dynamics of an individual driven vortex, which is especially interesting when pinning and elasticity compete. Furthermore, we establish a dragged vortex as a probe that extends deep into the bulk of the sample to interact with defects far from the surface, circumventing disadvantages of other local-probe techniques that give information only about the immediate vicinity of the surface ${ }^{8}$. Previous strategies for manipulating single vortices in superconductors usually applied forces relatively delocalized on the scale of $\lambda$ (refs 9-11). We use magnetic force microscopy (MFM) to combine imaging and vortex manipulation with a level of control far beyond what has been demonstrated before ${ }^{12,13}$. This capability may enable testing vortex entanglement ${ }^{14}$ and schemes for quantum computation ${ }^{15,16}$.

Our sample is ideal for studying the interplay between pinning and elasticity, with weak, well-controlled, pinning and fairly rigid vortices. In $\mathrm{YBa}_{2} \mathrm{Cu}_{3} \mathrm{O}_{6.991}$ ( $\mathrm{YBCO}$ ), superconductivity arises in $\mathrm{CuO}_{2}$ planes, parallel to the $a$ and $b$ axes, and in $\mathrm{Cu}-\mathrm{O}$ chains, along the $b$ axis. In pristine samples, such as ours, oxygen vacancies in the chains are the dominant source of pinning ${ }^{17-19}$. The orthorhombic crystal structure gives rise to penetration depth anisotropy, enabling us to determine the orientation of the crystal axes in situ (see Supplementary Information, Fig. S1). We mounted the platelet-shaped sample with the $c$ axis along $\hat{z}$ and the $a$ axis $\sim 9^{\circ}$ from $\hat{x}$ (Fig. 1a).

MFM uses a sharp magnetic tip on a flexible cantilever. Our tip magnetization provided an attractive tip-vortex force, $\mathbf{F}$. While rastering in the $x-y$ plane, parallel to the sample surface, we measured local variations in the resonant frequency of the cantilever to determine $\partial F_{z} / \partial z$ (ref. 20). We deliberately used the lateral components of $\mathbf{F}, F_{x} \hat{x}+F_{y} \hat{y} \equiv \mathbf{F}_{\text {lat }}$, for vortex manipulation.

At low temperature $(T \approx 5 \mathrm{~K})$, we observed no vortex motion up to our largest lateral force, $20 \mathrm{pN}$. At higher temperatures, pinning is reduced, and we could reduce the tip-sample distance $z$ to tune from non-invasive imaging to manipulation. Figure 1 shows typical examples for individual, well-isolated, vortices. The similarity of the behaviour of vortices pinned at different locations indicates the uniformity of the pinning landscape in this sample.

Figure 1d shows one of our two main findings: 'vortex wiggling'. Adding an alternating transverse force enhances vortex dragging markedly. The wiggling occurs because of the rastering associated with imaging: between incremental steps forward along the 'slow' scan direction, we raster the tip left and right along the 'fast' scan axis. If $F_{\text {lat }} \equiv\left|\mathbf{F}_{\text {lat }}\right|$ is small, this creates an image of a stationary vortex (Fig. 1a,c,e). When $F_{\text {lat }}$ is large, the vortex moves as the tip passes over it (Fig. 1b,d,f). Although this motion is substantial in the quasi-one-dimensional (1D) scans along the fast axis (Fig. 1f), it is much larger along the slow axis (Fig. 1d).

We study wiggling further in Fig. 2. Figure $2 \mathrm{a}$ shows a scan that we acquired after imaging the same area over and over, resulting in an enhanced wiggling effect. Line scans along $\pm \hat{x}$ from that image (Fig. 2b) do not differ from Fig. 1f. To study the vortex after completing the scan in Fig. 2a, we moved the tip back and forth over the centre of the vortex along $\pm \hat{y}$ to obtain Fig. $2 \mathrm{c}$,d. Details of the motion along the fast axis (Fig. 2b) are similar to motion along what was the slow axis (Fig. 2c,d) - a vortex moves in jerks, reminiscent of avalanches 5 - but the total distance moved differs substantially. Moreover, Fig. 2c,d shows that although a vortex moves very freely between the initial and final positions in Fig. 2a, it does not readily move outside this range. In fact, we were never able to permanently drag a vortex very far from its original location, contrary to the case in thin films ${ }^{12}$. This tethering suggests that each vortex was pinned along its full length across the $40-\mu \mathrm{m}$-thick crystal, and that we observed the vortex stretching.

A vortex presumably stops moving where elasticity and pinning balance $F_{\text {lat }}$. Qualitatively, wiggling helps segments of the vortex to depin, facilitating the extra motion along the slow axis. Confirming this is Fig. 2 with the enhancement of the effect by

\footnotetext{
${ }^{1}$ Geballe Laboratory for Advanced Materials, Stanford University, Stanford, California 94305, USA, ${ }^{2}$ Department of Physics, Harvard University, Cambridge, Massachusetts 02138, USA, ${ }^{3}$ Department of Condensed Matter Physics, Weizmann Institute of Science, Rehovot 76100, Israel, ${ }^{4}$ Department of Physics and Astronomy, University of British Columbia, Vancouver, British Columbia V6T 1Z1, Canada. *e-mail: ophir@stanford.edu.
} 


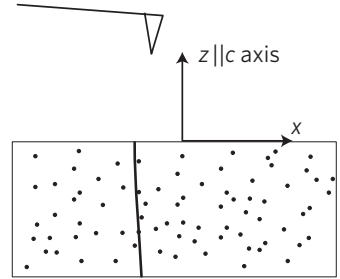

c

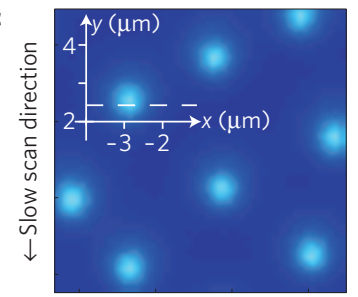

Fast scan direction $\rightarrow$

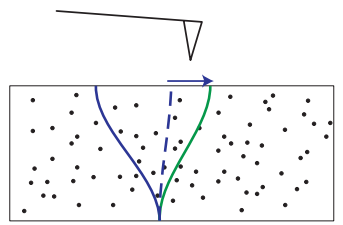

d

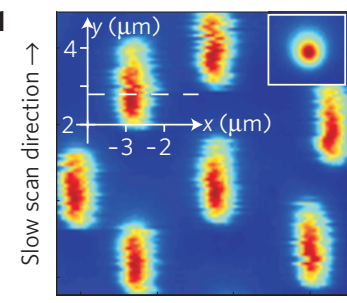

Fast scan direction $\rightarrow$
Span:140 pN $\mu m^{-1}$

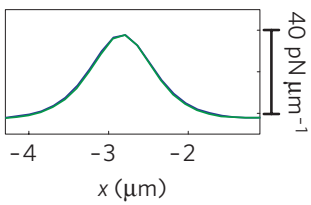

$\mathbf{f}$

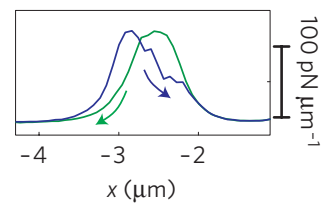

Figure 1 | MFM imaging and manipulation of individual vortices in YBCO at $\mathbf{T}=\mathbf{2 2 . 3} \mathbf{K}$. a,b, Schematic diagrams, not drawn to scale, showing an MFM tip (triangles) that attracts a vortex (thick lines) in a sample with randomly distributed point pinning sites (dots): at 'surveillance' height, the applied force $F_{\text {lat }}$ is too weak to move the vortex (a); at manipulation height the vortex moves right and then left, as the tip rasters over it (b). Here, we illustrate what happens in a scan along $+\hat{x}$, as indicated by the arrow. Also shown are three configurations of the vortex, previously dragged along $-\hat{x}$ : the blue line on the left illustrates the vortex before the tip drags it to the right, the dashed blue line shows an intermediate configuration as it follows the tip and the green line on the right shows the final configuration, after the tip moves away. c,d, MFM scans for two different scan heights (colour scale gives $\partial F_{z} / \partial z$; fast scan and slow scan directions are indicated on the frame): $z=420 \mathrm{~nm}$ (maximum applied lateral force $F_{\text {lat }}^{\max } \approx 6 \mathrm{pN}$ ), not low enough to perturb vortices at this temperature $(\mathbf{c}) ; z=170 \mathrm{~nm}$

$\left(F_{\text {lat }}^{\max } \approx 12 \mathrm{pN}\right.$ ), low enough to drag the vortices significantly (d). Inset: Scan at $5.2 \mathrm{~K}$, showing a stationary vortex at tip height and force comparable to those in the main panel. e, Line cut through the data in c along the dashed line, showing the signal from a stationary vortex (blue). Overlapping it is a line cut from the reverse scan (green). $\mathbf{f}$, Line cut through the data in $\mathbf{d}$ along the dashed line, showing a typical signal from a dragged vortex. The right arrow shows the data acquired with the tip moving along $+\hat{x}$, as in $\mathbf{d}$ the left arrow shows the data acquired with the tip moving back along $-\hat{x}$.

adding wiggling cycles (further test in Supplementary Information, Fig. S2). Wiggling is reminiscent of 'vortex shaking', used to accelerate equilibration in vortex matter by oscillating a magnetic field perpendicular to the applied magnetic field generating the vortices $^{6,21,22}$, and may be the mechanism for it. The models presented below describe motion without wiggling, as we observe along the fast axis. Our single-vortex data should be amenable to more advanced and quantitative theoretical modelling to describe the wiggling.

The behaviour along the fast axis can be analysed as individual one-dimensional scans. As the tip approaches a vortex, $F_{\text {lat }}$ increases until, if it overcomes pinning, the vortex moves. The vortex then

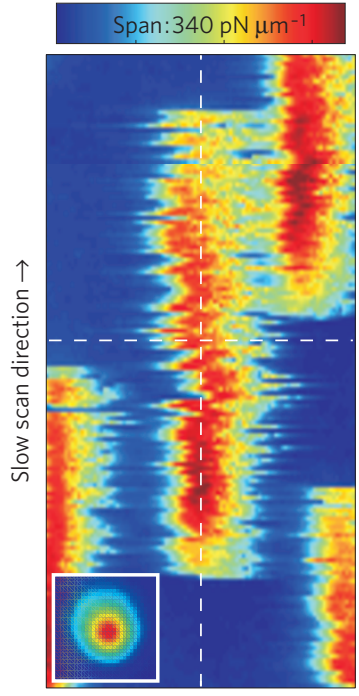

Fast scan direction $\rightarrow$

b

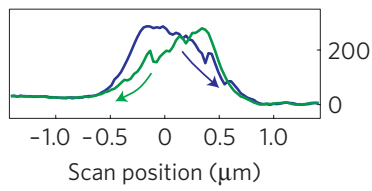

Figure 2 | MFM image and line scans at $\mathbf{T}=\mathbf{2 0} \mathrm{K}$, showing how wiggling enhances dragging along the slow direction. $\mathbf{a}$, Scan at $z=80 \mathrm{~nm}$

$\left(F_{\text {lat }}^{\max } \approx 20 \mathrm{pN}\right.$ ), acquired after repeated imaging, reversing the slow scan direction for each new scan, which enhances the wiggling effect. Scan directions are denoted on the frame; dashed lines show the trajectories of the line scans in b-d. Inset: Scan with similar parameters at $5.2 \mathrm{~K}$, where vortices were immobile. $\mathbf{b}$, Line scans from a along the horizontal dashed line. Arrows show the scan direction. c,d, Immediately after the scan in $\mathbf{a}$, we scanned back and forth along the vertical dashed line at $z=80 \mathrm{~nm}$ ((c) scans down, (d) scans up). Indices: Order of line-scan acquisition.

moves until pinning and the growing elastic force balance $F_{\text {lat }}$, presumably when $F_{\text {lat }}$ is maximal, enabling us to treat this as a static problem. The dependence of $w$, the distance dragged along the fast axis, on $F_{\text {lat }}^{\max } \equiv \max \left(F_{\text {lat }}\right)$ is shown in Fig. 3. We have constructed a model based on weak collective pinning (WCP) for a single vortex ${ }^{1}$ to explain this data (see Supplementary Information, Discussion S2). WCP assumes that pinning is only by the collective, cumulative, effect of many pinning sites, each too weak to pin a vortex on its own, and that vortices are elastic strings ${ }^{23}$, as described in Ginzburg-Landau theory ${ }^{1}$. A characteristic length, $L_{\mathrm{c}}$, emerges from the competition between pinning and elasticity: a vortex takes advantage of pinning by bending on a length $L \gg L_{\mathrm{c}}$, but it cannot bend for $L \ll L_{\mathrm{c}}$. In WCP, the vortex is broken into elastically coupled $L_{\mathrm{c}}$ segments, each pinned by a characteristic force, $F_{\mathrm{p}}$.

We assume that each vortex is initially along the $c$ axis, that only the top portion interacts directly with the tip and that $L_{\mathrm{c}} \ll \lambda_{a b}$ (where $\lambda_{a b}$ is the in-plane penetration depth). Each $L_{c}$ segment is subject to elastic forces from neighbouring segments and a pinning force up to $F_{\mathrm{p}}$, modelled as static friction. We find that the top portion of a vortex depins when $F_{\text {lat }}^{\max }>F_{\mathrm{p}} \Lambda / L_{\mathrm{c}}\left(\Lambda\right.$ of order $\left.\lambda_{a b}\right)$ and that:

$$
w \sim \frac{F_{\text {lat }}^{\max }\left(F_{\text {lat }}^{\max }-F_{1}\right)}{2 F_{\mathrm{p}} \varepsilon_{\perp} / L_{\mathrm{c}}},
$$

for $F_{\text {lat }}^{\max } \gg F_{\mathrm{p}} \Lambda / L_{\mathrm{c}}$ (see Supplementary Information, Discussion S3). Here, $F_{1} / F_{\mathrm{p}} \approx 2 \Lambda / L_{\mathrm{c}}$ and $\varepsilon_{\perp}$ is the vortex line tension. 


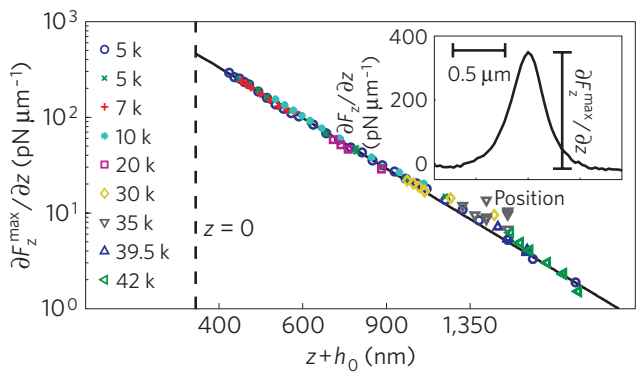

b

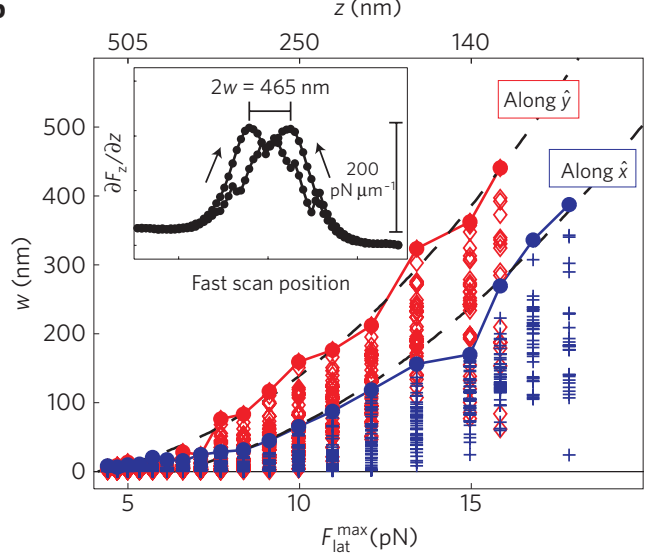

Figure 3 | Force dependence of the dragged distance, $\boldsymbol{w}$, along the fast axis. $\mathbf{a}$, Force calibration: magnitude of peaks from vortices, $\max \left(\partial F_{z} / \partial z\right) \equiv \partial F_{z}^{\max } / \partial z$, versus scan height at various temperatures. Clearly the temperature dependence in this range is weak. The solid line is a fit to $\max \left(\partial F_{z} / \partial z\right)=\left(\tilde{m} \Phi_{0} / \pi\right) /\left(z+h_{0}\right)^{3}\left(h_{0}=358 \pm 5 \mathrm{~nm}, \tilde{m}=32 \pm 1 \mathrm{nAm}\right.$, Supplementary Information, Discussion S1). The excellent fit enables us to use $F_{z}^{\max }=\left(\tilde{m} \Phi_{0} / 2 \pi\right) /\left(z+h_{0}\right)^{2}$. The maximum applied lateral force is given by $F_{\text {lat }}^{\max }=\alpha F_{z}^{\max }$. For a wide range of tip shapes $0.3<\alpha<0.4$ (ref. 30). We set $\alpha=0.35$, adding at most $25 \%$ systematic error to $F_{\text {lat }}^{\max }$. Inset: Single line from a scan at $z=65 \mathrm{~nm}, T=5.2 \mathrm{~K}$, showing the peak height for an immobile vortex (here, $\left.\max \left(\partial F_{z} / \partial z\right)=365 \mathrm{pN} \mu \mathrm{m}^{-1}\right)$. b. Distance moved by the vortex along the fast direction versus $z$ (top axis) acquired at $T=25 \mathrm{~K}$ versus $F_{\text {lat }}^{\text {max }}$ (bottom axis). In addition to the maximum $w$ (filled circles), we plot the distribution (diamonds and crosses), which shows the stochasticity of the vortex motion. Other errors are not shown. Dashed lines are fits to equation (1) (along $\hat{x}: F_{1}=6.3 \pm 0.4 \mathrm{pN}, F_{\mathrm{p}} \varepsilon_{\perp} / L_{\mathrm{c}}=272 \pm 12 p \mathrm{~N}^{2} \mu \mathrm{m}^{-1}$; along $\hat{y}$ : $F_{1}=4.1 \pm 0.7 \mathrm{pN}, F_{\mathrm{p}} \varepsilon_{\perp} / L_{\mathrm{c}}=212 \pm 17 \mathrm{pN}^{2} \mu \mathrm{m}^{-1}$ ). Inset: Subsequent line scans showing the jerky nature of the vortex motion and how $w$ was extracted from the difference of the vortex positions in pairs of subsequent line scans.

a

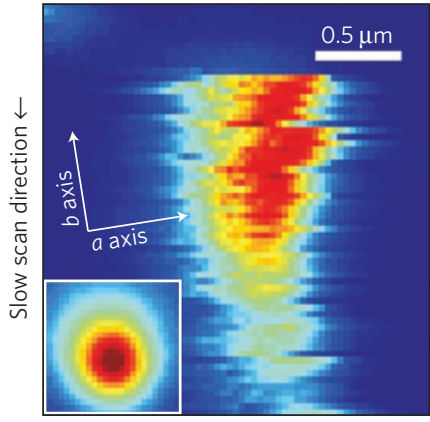

Fast scan direction $\leftarrow$

c

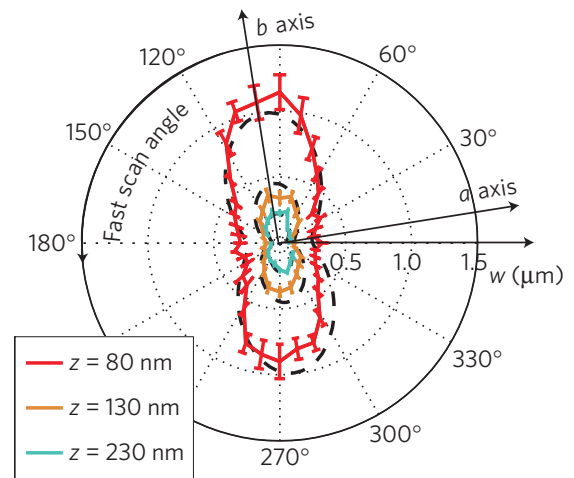

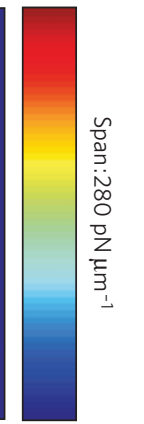

d
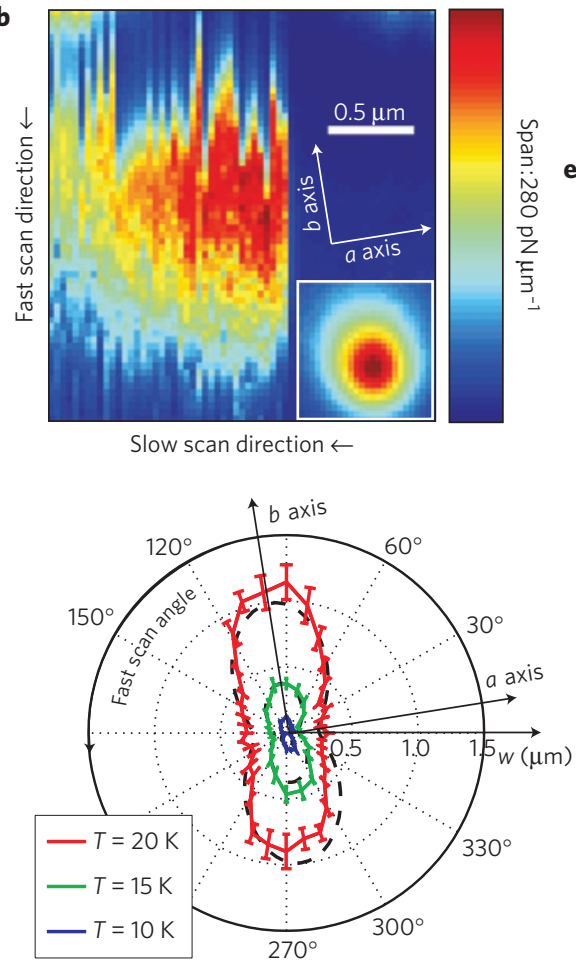

Slow scan direction $\leftarrow$

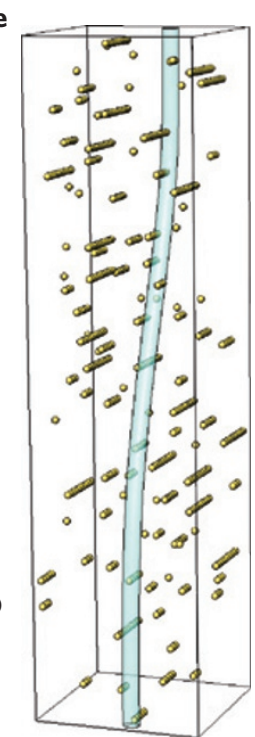

Figure 4 | Images and analysis showing the anisotropy of dragging when the fast axis is along the $a$ axis and when it is along the $b$ axis. $a, b, V o r t e x$ motion when the fast scan is along $\hat{x}(\mathbf{a})$ and $\hat{y}(\mathbf{b})\left(T=20 \mathrm{~K}, z=80 \mathrm{~nm}, F_{\text {lat }}^{\max } \approx 20 \mathrm{pN}\right)$. Note the erratic nature of the motion and the abrupt snap-in to the tip on approaching the vortex along the slow axis, apparent as a sharp onset of the signal. Insets: Images of immobile vortices obtained with the same tip, scan height and scan directions at $T=5.2 \mathrm{~K}$. c,d, Distance moved along the fast axis, $w$, measured near the maximum lateral force, versus scan angle measured from $\hat{x}$. Bars denote $70 \%$ confidence intervals. Dashed lines show the fit described in the text, with the oxygen vacancy cluster size $2 R_{b}$ as a free parameter. Fit result: $2 R_{b} / \xi_{a b}=0.7$. In c, $T=20 \mathrm{~K}$ at $z=80 \mathrm{~nm}, 130 \mathrm{~nm}, 230 \mathrm{~nm}\left(F_{\text {lat }}^{\max } \approx 20,15,10 \mathrm{pN}\right.$, respectively). In d, $z=80 \mathrm{~nm}$, for $T=20,15,10 \mathrm{~K}$. e, Schematic diagram of vortex core meandering across the crystal in the presence of point defects clustered along the $b$ axis. 
Figure $3 \mathrm{~b}$ shows a reasonable fit for large $F_{\mathrm{lat}}^{\max }$, where the exact shape of the top portion of the vortex and the fine details of the pinning landscape are not important.

Figure $3 \mathrm{~b}$ also highlights our second main finding-in-plane anisotropy due to the crystal: we can drag a vortex farther along the fast axis if it is along the YBCO $b$ axis than if it is along the $a$ axis. This is also apparent in Fig. 4. Figure 4c,d shows the dependence of $w$ on the fast scan angle (see Supplementary Information, Fig. S3, for $w$ here). We found a similar effect using different tips (see Supplementary Information, Table S1) and for every vortex that we probed.

Equation (1) explains the anisotropy qualitatively. The penetration depth imparts in-plane anisotropy to $\varepsilon_{\perp}$ (refs 1,24), implying that it is easier to tilt a vortex towards the $b$ axis. In addition, because of the in-plane anisotropy of the vortex core radius, even for point pinning, the effective pinning potential is less steep along the $b$ axis, implying a smaller depinning force. Equation (1) thus naturally explains the weak dependence of the anisotropy on $F_{\text {lat }}^{\max }$ and $T$, seen in Fig. 4c,d. When $F_{\text {lat }}^{\max } \gg F_{1}$, the only dependence on $F_{\text {lat }}^{\max }$ is an overall scale. The same is true for the $T$ dependence, because both the superconducting parameters and the defect structure depend only weakly on $T$ in our range $\mathrm{e}^{25}$.

For quantitative analysis, we have determined the angular dependence of $w \propto L_{\mathrm{c}} / \varepsilon_{\perp} F_{\mathrm{p}}$ (see Supplementary Information, Discussion S4), which depends on the anisotropy factor $\zeta$. Fitting all five data sets in Fig. 4c,d, we find $\zeta=1.6$ (see Supplementary Information, Fig. S5), in clear disagreement with our direct measurement (see Supplementary Information, Fig. S1) and the known value ${ }^{25}, \zeta=1.3$. We surmise that there is an extra source of anisotropy.

A likely source for extra pinning anisotropy is nanoscale clustering of oxygen vacancies along the $\mathrm{Cu}-\mathrm{O}$ chains ${ }^{26,27}$ (Fig. 4e). We modified our model (see Supplementary Information, Discussion S5), imposing $\zeta=1.3$, and fit the data in Fig. $4 \mathrm{c}, \mathrm{d}$ for the cluster size. We find clusters of order 10 vacancies along the $\mathrm{Cu}-\mathrm{O}$ chains (see Supplementary Information, Discussion S5), large but reasonable for the sample's length of time at room temperature, where oxygen vacancies migrate slowly and cluster ${ }^{28}$. It is also possible that non-Ginzburg-Landau physics affects the core structure $^{29}$, changing details of the pinning-force anisotropy. These results demonstrate that single-vortex manipulation is a local probe of both the core and the defect structure on a scale down to the core size. For example, in samples without intrinsic $a-b$ anisotropy and with known defect structure, MFM could be used to probe the intrinsic structure of the vortex core itself.

Despite the fact that YBCO is one of the most studied superconductors, our data reveal major surprises about the behaviour of individual vortices. A model based on weak collective pinning for a single vortex quantitatively describes quasistatic aspects of vortex motion with the incorporation of anisotropy in the local microscopic pinning. This demonstrates that singlevortex manipulation is a local probe of the structure of both the vortex and the pinning defects. Further work is required to describe the dynamic aspects of individual vortex motion that we revealed: its stochastic nature and the marked effect of transverse wiggling. In particular: how do the dynamics alter the effective pinning landscape? How do they affect the mechanical properties? Practically, wiggling is an important tool for future experiments that require pulling vortices long distances, for example, in the study of vortex entanglement ${ }^{14}$. Our results show the utility of local force probes for accessing the pinning properties and mechanical behaviour of individual vortices, the collective behaviour of which is of great importance for the properties of superconductors.

\section{Methods}

Frequency-modulated MFM (ref. 20). We oscillate the cantilever along $\hat{z}$ and measure the resonant frequency, $f_{0}$, which shifts by $\Delta f$ in a force gradient. Assuming small oscillation amplitude, $\partial F_{z} / \partial z \approx-2 c \Delta f / f_{0}$ (where $c$ is the cantilever spring constant, see Supplementary Information, Table S1).

Sample details. The platelet-shaped single crystal was grown from flux in a $\mathrm{BaZrO}_{3}$ crucible for high purity and crystallinity ${ }^{17}$. The (001) surfaces were free of visible inclusions. Mechanical detwinning was followed by annealing to oxygen content $7-\delta=6.991$, implying $T_{\mathrm{c}} \approx 88 \mathrm{~K}$ (ref. 19). The sample was stored at room temperature for a few years. The finite $a-b$ anisotropy enabled us to identify crystal orientation by determining the directions along which vortex spacings were extremal (see Supplementary Information, Fig. S1). The ratio between these spacings is $\zeta=1.3$, corroborating previous results on similar samples ${ }^{25}$.

Received 4 July 2008; accepted 16 September 2008; published online 16 November 2008

\section{References}

1. Blatter, G., Feigel'man, M. V., Geshkenbein, V. B., Larkin, A. I. \& Vinokur, V. M. Vortices in high-temperature superconductors. Rev. Mod. Phys. 66, 1125-1388 (1994).

2. Fisher, M. P. A. \& Lee, D. H. Correspondence between two-dimensional bosons and a bulk superconductor in a magnetic field. Phys. Rev. B 39, 2756-2759 (1989).

3. Nelson, D. R. \& Vinokur, V. M. Boson localization and correlated pinning of superconducting vortex arrays. Phys. Rev. B 48, 13060-13097 (1993).

4. Ertaş, D. \& Kardar, M. Anisotropic scaling in depinning of a flux line. Phys. Rev. Lett. 73, 1703-1706 (1994).

5. Sethna, J. P., Dahmen, K. A. \& Myers, C. R. Crackling noise. Nature 410, 242-250 (2001)

6. Avraham, N. et al. 'Inverse' melting of a vortex lattice. Nature 411, 451-454 (2001).

7. Pardo, F., de la Cruz, F., Gammel, P. L., Bucher, E. \& Bishop, D. J. Observation of smectic and moving-Bragg-glass phases in flowing vortex lattices. Nature 396, 348-350 (1998).

8. Hoffman, J. E. et al. Imaging quasiparticle interference in $\mathrm{Bi}_{2} \mathrm{Sr}_{2} \mathrm{CaCu}_{2} \mathrm{O}_{8+\delta}$. Science 297, 1148-1151 (2002).

9. Park, G. S., Cunningham, C. E., Cabrera, B. \& Huber, M. E. Vortex pinning force in a superconducting niobium strip. Phys. Rev. Lett. 68, 1920-1922 (1992).

10. Breitwisch, M. \& Finnemore, D. K. Pinning of a single Abrikosov vortex in superconducting $\mathrm{Nb}$ thin films using artificially induced pinning sites. Phys. Rev. B 62, 671-677 (2000).

11. Gardner, B. W. et al. Manipulation of single vortices in $\mathrm{YBa}_{2} \mathrm{Cu}_{3} \mathrm{O}_{6.354}$ with a locally applied magnetic field. Appl. Phys. Lett. 80, 1010-1012 (2002).

12. Moser, A., Hug, H. J., Stiefel, B. \& Güntherodt, H.-J. Low temperature magnetic force microscopy on $\mathrm{YBa}_{2} \mathrm{Cu}_{3} \mathrm{O}_{7-\delta}$ thin films. J. Magn. Magn. Mater. 190, 114-123 (1998).

13. Roseman, M. \& Grütter, P. Magnetic imaging and dissipation force microscopy of vortices on superconducting Nb films. Appl. Surf. Sci. 188, 416-420 (2002).

14. Olson Reichhardt, C. J. \& Hastings, M. B. Do vortices entangle? Phys. Rev. Lett. 92, 157002 (2004).

15. Berciu, M., Rappoport, T. G. \& Boldizsár, J. Manipulating spin and charge in magnetic semiconductors using superconducting vortices. Nature 435, 71-75 (2005).

16. Weeks, C., Rosenberg, G., Seradjeh, B. \& Franz, M. Anyons in a weakly interacting system. Nature Phys. 3, 796-801 (2007).

17. Liang, R., Bonn, D. A. \& Hardy, W. N. Growth of high quality YBCO single crystals using $\mathrm{BaZrO}_{3}$ crucibles. Physica C 304, 105-111 (1998).

18. Liang, R. X., Bonn, D. A. \& Hardy, W. N. Preparation and X-ray characterization of highly ordered ortho-II phase $\mathrm{YBa}_{2} \mathrm{Cu}_{3} \mathrm{O}_{6.50}$ single crystals. Physica C 336, 57-62 (2000).

19. Liang, R., Bonn, D. A. \& Hardy, W. N. Evaluation of $\mathrm{CuO}_{2}$ plane hole doping in $\mathrm{YBa}_{2} \mathrm{Cu}_{3} \mathrm{O}_{6+x}$ single crystals. Phys. Rev. B 73, 180505 (2006)

20. Albrecht, T. R., Grütter, P., Horne, D. \& Rugar, D. Frequency-modulation detection using high-Q cantilevers for enhanced force microscope sensitivity. J. Appl. Phys. 69, 668-673 (1991).

21. Willemin, M. et al. Strong shift of the irreversibility line in high- $T_{\mathrm{c}}$ superconductors upon vortex shaking with an oscillating magnetic field. Phys. Rev. B 58, R5940-R5943 (1998).

22. Brandt, E. H. \& Mikitik, G. P. Why an ac magnetic field shifts the irreversibility line in type-II superconductors. Phys. Rev. Lett. 89, 027002 (2002).

23. Brandt, E. H. Large range of validity of linear elasticity of the vortex lattice in high- $T_{\mathrm{c}}$ superconductors. Phys. Rev. Lett. 69, 1105-1108 (1992).

24. Burlachkov, L. I. Structure of vortex lattice in biaxial superconductors. Europhys. Lett. 8, 673-677 (1989). 
25. Pereg-Barnea, T. et al. Absolute values of the London penetration depth in $\mathrm{YBa}_{2} \mathrm{Cu}_{3} \mathrm{O}_{6+y}$ measured by zero field ESR spectroscopy on Gd doped single crystals. Phys. Rev. B 69, 184513 (2004).

26. Erb, A. et al. Experimental evidence for fast cluster formation of chain oxygen vacancies in $\mathrm{YBa}_{2} \mathrm{Cu}_{3} \mathrm{O}_{7-\delta}$ as the origin of the fishtail anomaly. Solid State Commun. 112, 245-249 (1999).

27. Nishizaki, T., Shibata, K., Maki, M. \& Kobayashi, N Vortex phase transition and oxygen vacancy in $\mathrm{YBa}_{2} \mathrm{Cu}_{3} \mathrm{O}_{y}$ single crystals. J. Low Temp. Phys. 131, 931-940 (2003).

28. Liang, R. et al. Preparation and characterization of homogeneous YBCO single crystals with doping level near the SC-AFM boundary. Physica C 383, $1-7$ (2002)

29. Sonier, J. E. et al. Expansion of the vortex cores in $\mathrm{YBa}_{2} \mathrm{Cu}_{3} \mathrm{O}_{6.95}$ at low magnetic fields. Phys. Rev. B 59, R729-R732 (1999).

30. Wadas, A., Fritz, O., Hug, H. J. \& Güntherodt, H.-J. Magnetic force microscopy signal of flux line above a semiinfinite

type-II-superconductor-theoretical approach. Z. Phys. B 88, 317-320 (1992).

\section{Acknowledgements}

Research supported by DOE Contract No. DE-AC02-76SF00515, AFOSR grant FA50-05-1-0290, the Packard Foundation and the US-Israel Binational Science Foundation (BSF). The experimental set up was developed with extensive help from D. Rugar. We acknowledge useful discussions with J. R. Kirtley, H. Bluhm, G. P. Mikitik, E. H. Brandt and V. B. Geshkenbein.

\section{Author contributions}

Conception and design: O.M.A., L.L., E.W.J.S., J.E.H., N.C.K. and K.A.M. Experiments: O.M.A. and L.L. Analysis: O.M.A. and L.L. with ideas developed with E.Z. and K.A.M. Sample growth: D.A.B., R.L. and W.N.H. Manuscript preparation: O.M.A., L.L., E.Z. and K.A.M., with input from all co-authors.

\section{Additional information}

Supplementary Information accompanies this paper on www.nature.com/naturephysics. Reprints and permissions information is available online at http://npg.nature.com/ reprintsandpermissions. Correspondence and requests for materials should be addressed to O.M.A. 